\title{
FRONTAL, CORONAL AND SAGITTAL PLANE VECTORCARDIO- GRAPHY WITH A TWO-CHANNEL SCALAR ELECTROCARDIOGRAPH
}

\author{
BY \\ E. J. FISCHMANN \\ with an appendix by \\ D. BROWN \\ From the Cardiology Department, Green Lane Hospital, Auckland and the Department of Physics, \\ Auckland University College, New Zealand. \\ Received April 15, 1954
}

A method of three-dimensional vectorcardiography will be described. It appears to yield much of the information supplied by the cathode-ray vectorcardiograph, without being as cumbersome and costly. It uses scalar leads, but is free of those inaccuracies (Graybiel and White, 1952) of Grant and Estes' (1951) method, which are caused by asynchronous recording of leads and by the proximity of electrodes to the heart.

\section{METHOD OF RECORDING}

An Elmquvist Junior electrocardiograph with two galvanometers and a second amplifier was used. Calibration was either one and a half times or twice the usual $(1 \mathrm{mV}=1 \mathrm{~cm}$.) paper speed approximately $5 \mathrm{~cm}$. per sec. A " cube" reference system was used, with a modification (Grishman et al, 1951) of Duchosal and Sulzer's (1949) lead placement and, except that the polarity of the sagittal lead was reversed for sagittal plane recording, with the lead connections advocated by Shillingford and Brigden (1951).

Leads were called $\mathrm{X}$ (transverse), $\mathrm{Y}$ (caudo-cranial), and $\mathrm{Z}$ (postero-anterior). $\mathrm{Y}$ and $\mathrm{X}$ were recorded synchronously to determine frontal, $\mathrm{Z}$ and $\mathrm{X}$ coronal, $\mathrm{Y}$ and $\mathrm{Z}$ sagittal plane vectors. The frontal plane was "viewed" from the front, the coronal from below, the sagittal from the left. Thus "viewed" in each plane the first-named lead appeared as the "vertical," the second as the " horizontal " component lead (Fig. 1A, B and Fig. 2: corner insets). " Vertical " leads appeared in the upper, "horizontal" leads in the lower tracing of each cardiograph. In the main paper the orientation of vectors was stated in terms of the Einthoven convention (as viewed by the observer $0^{\circ}$ right, $90^{\circ}$ bottom, $180^{\circ}$ left, $-90^{\circ}$ top of the page; quadrants numbered counterclockwise, commencing with the $0^{\circ}$ to $90^{\circ}$ quadrant). In the appendix, to facilitate mathematical treatment, the notation traditional in physics was used $\left(90^{\circ}\right.$ top, $-90^{\circ}$ bottom; quadrants clockwise numbered, commencing with what would be the fourth quadrant of the former notation).

Lead-pair records in three planes were obtained from each of 100 subjects without, and 147 subjects with clinical and/or electrocardiographic evidence of heart disease.

\section{Construction of the frontal, Coronal, and Sagittal Plane Projections of Spatial INSTANTANEOUS VECTORS}

Frontal plane. The heart's electromotive force, having direction as well as magnitude, is a yector quantity and can be described by a directed line called the spatial instantaneous vector. Its frontal plane projection is the frontal plane instantaneous vector $\mathrm{Ef}$. At a given instant, $y$ and $x$, the potential differences 

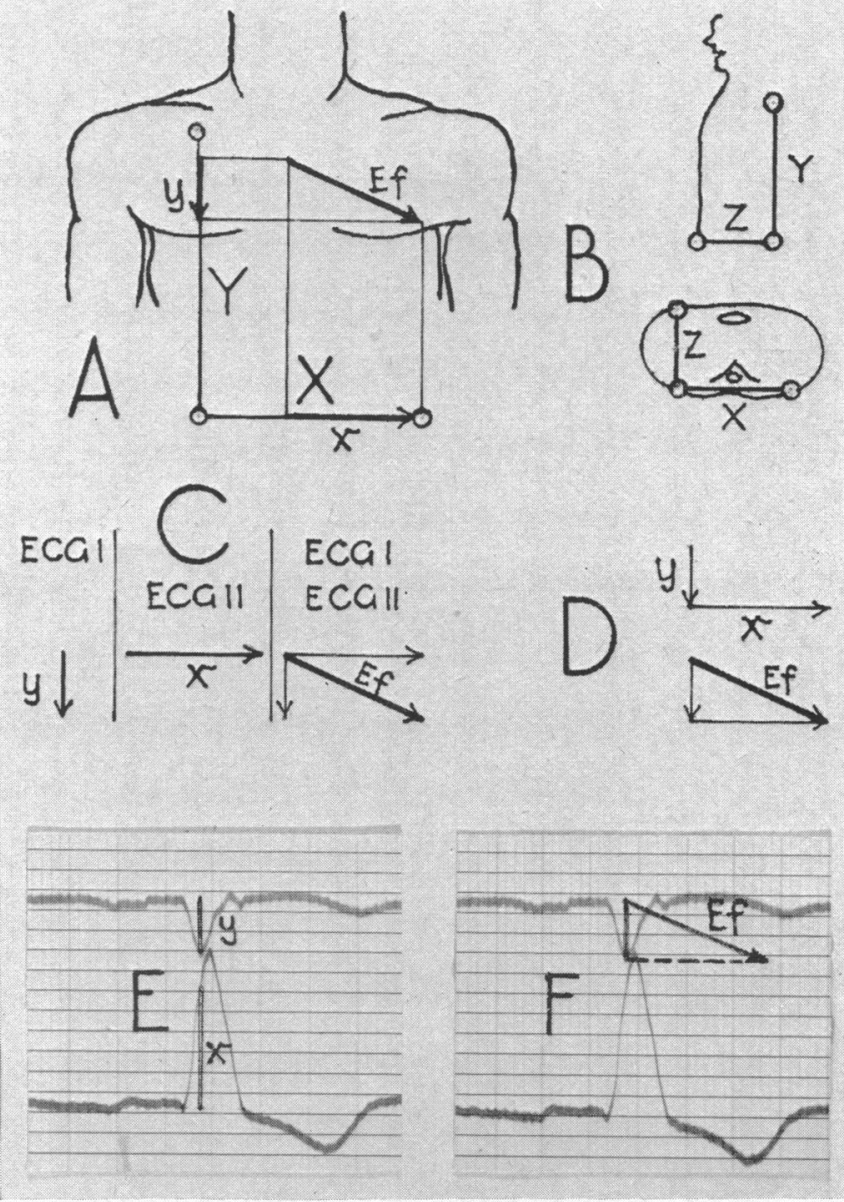

Fig. 1.-(A) Caudo-cranial lead Y, transverse lead X, and frontal plane instantaneous vector Ef; $y$ and $x$, the synchronous potential differences in the two leads, are "vertical" and "horizontal" components of Ef. (B) Postero-anterior lead $Z$ is the "vertical"; $X$ the " horizontal " lead in the coronal; $Y$ the "vertical " $Z$ the " horizontal " lead in the sagittal plane.

Summation $\mathrm{y}+\mathrm{x}=E f$ : (C) By electronic vectorcardiograph. (D) By setting $x$ off from end of $y$ and joining free onset to free end. (E) On scalar ECG strip: $y$ and $x$ at $0.04 \mathrm{sec}$., recorded as vertical deflections. (F) Clockwise tilted lower lead component $x$ (dotted line) set off from end of synchronous upper lead component; Ef joins free onset to free end. Time marks $1 / 10$ and $1 / 50 \mathrm{sec}$.

along leads $Y$ and $X$ are vertical and horizontal components of Ef (Fig. 1A). Therefore, if $y$ and $x$ are known, Ef can be determined. Determining a resultant vector from its components is known as the summation of components. According to the "parallelogram law" the sum is the diagonal of a parallelogram of which the components are sides. The cathode-ray vectorcardiograph performs the summation, according to this law, of components supplied by two cardiograph channels. In contrast to the conventional electrocardiograph, which records vertical movement alone, the beam of the cathode-ray vectorcardiograph will also travel horizontally or obliquely. If one of its channels gave a vertical deflection $y$, and the other a horizontal deflection $x$, the two channels together applied would produce a deflection corresponding to $\mathrm{Ef}$, the parallelogram diagonal (Fig. 1C).

If leads $\mathbf{Y}$ and $\mathbf{X}$ are simultaneously recorded with a two-channel conventional electrocardiograph, 
beam or (string) excursions at a given instant will equal $y$ and $x$ in magnitude, but not in direction. As vertical movement alone is possible, the horizontal $x$ (lower lead) component will also appear vertically orientated (Fig. 1E). It must therefore be tilted by $90^{\circ}$. With the present lead connections the tilt must be clockwise (Fig. 1F). Summation of $y$ and of clockwise tilted $x$ will then give Ef. The components $x$ and $y$ must be taken from synchronous points of the two tracings.

A simplified method of vector summation, commonly used in vector algebra (Henrici and Turner, 1903; Rutherford, 1951) was found more convenient for the present purpose than parallelogram construction, although in essence identical with the latter (cf. Fig. 1C and D). Expressed in the terms here used, if $\mathrm{x}$ is set off from the distal end of $\mathrm{y}, E f$ is the straight line directed from free onset to free end (Fig. 1D). Three ways of using this principle in the construction of cardiac instantaneous vectors are offered in the following.

(1) Drawing on the cardiogram strip, or visually with the aid of the coordinate system formed by the time and voltage lines, the clockwise tilted lower lead deflection $x$ is transferred to the distal end of $y$. Ef is the straight line connecting free onset with free end (Fig. 1E, F).

(2) Main Fig. 2 shows a " biaxial " modification of Wenkebach and Winterberg's (1927) triaxial diagram

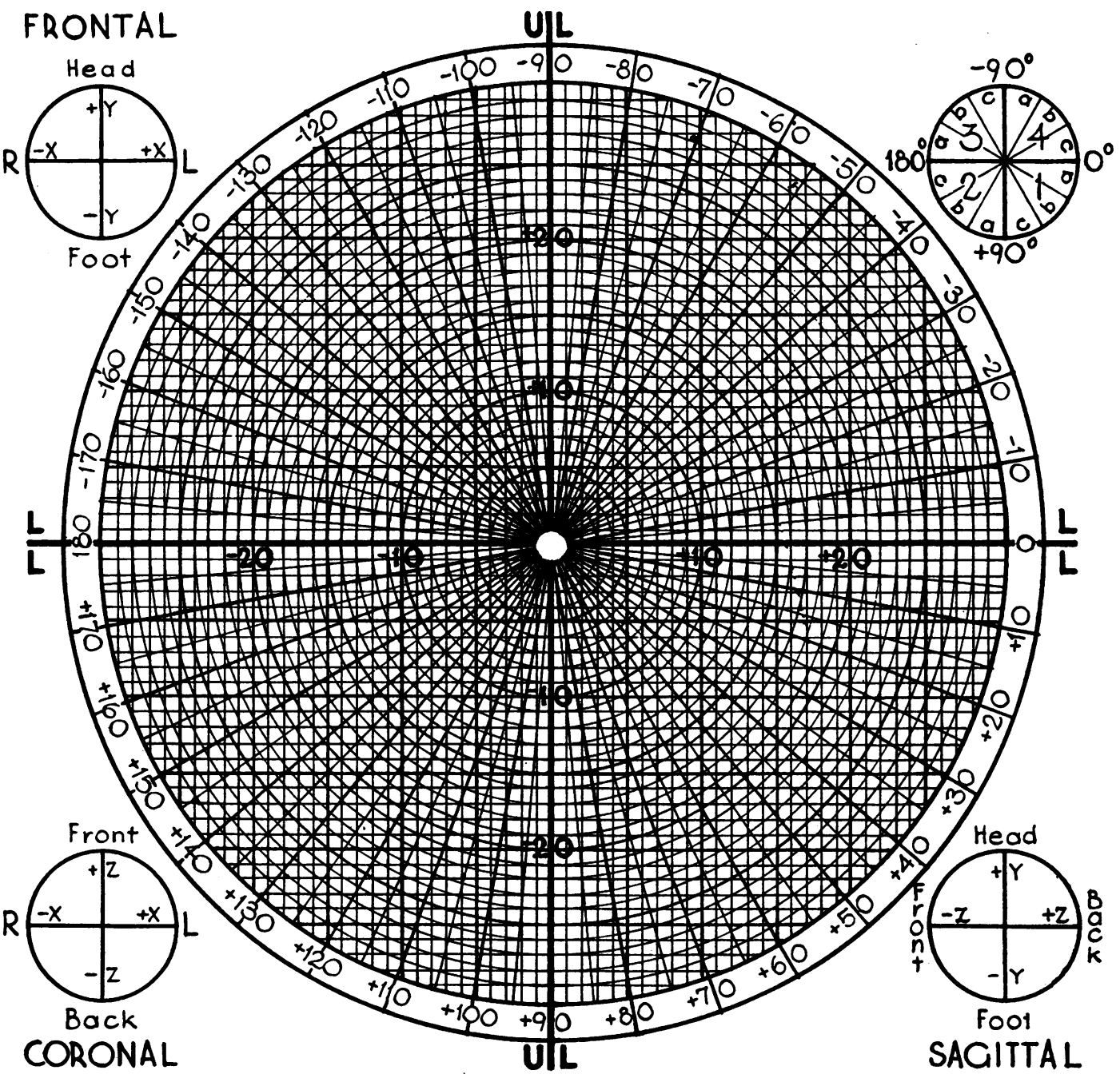

FIG. 2.-Biaxial chart, for the summation of vectors enclosing a right angle (after Wenkebach and Winterberg). UL axis of upper, LL of lower lead of a lead-pair. Positive half of axes corresponds to upward, negative half to downward component lead deflection; grading of axes to electrocardiographic amplitude in mm. Right upper inset: 4 quadrants and 12 sectors of reference system. Remaining three insets: topography of up- or downward component lead deflections in three planes. 
for the estimation of frontal plane vectors from standard leads. In Fig. $1 \mathrm{E}$ at $0.04 \mathrm{sec} ., y$ is $-6, x$ is $14 \mathrm{~mm}$. To determine Ef at $0.04 \mathrm{sec}$., -6 is found on the vertical axis of the diagram. The terminus of Ef is 14 units to the right of this point. It lies on the fifteenth of the concentric circles; the length of Ef is therefore $15 \mathrm{~mm}$. Its direction read at the border of the figure is $22 \cdot 5^{\circ}$.

(3) For approximate determination of direction, without magnitude, the quadrant containing Ef can be determined. The quadrant in question is that enclosed by the upper and the clockwise tilted lower lead component. Thus in Fig. 4B in each of 6 frontal plane lead pairs, $y$ at the instant corresponding to the tallest peak, points towards $90^{\circ}$; clockwise tilted $x$, points towards $0^{\circ}$; the two enclose the first quadrant. To define orientation of Ef further, each quadrant can then be divided into three $30^{\circ}$ " sectors " (Holzmann, 1945; Graettinger et. al., 1951; Lepeschkin, 1951) and for the present purpose sectors within each quadrant were lettered $a, b, c$, in clockwise order (Fig. 2, right upper inset).

In any quadrant, if the ratio of component $y$ to component $x$ is $6 / 10$ or roughly $2 / 3$, Ef diverges from the $\mathrm{X}$ axis, if it is $10 / 6$ from the $\mathrm{Y}$ axis, by $30^{\circ}$. This may be confirmed from main Fig. 2. Thus if the difference between the two components is less than 1/3, Ef is in sector b (Fig. 4B, third and fourth records); if greater than $1 / 3, \mathrm{Ef}$ is in the sector adjoining the axis of the lead with the taller component. In the first two records of Fig. 4B Ef is in sector a, adjoining the $\mathrm{X}$ axis; in the last two records Ef is in sector $\mathrm{c}$, adjoining the $\mathrm{Y}$ axis.

Frontal and Sagittal Planes. Replacing leads $\mathrm{Y}$ and $\mathrm{X}$ by $\mathrm{Z}$ and $\mathrm{X}$, or $\mathrm{Y}$ and $\mathrm{Z}$ (Fig. 1B), the coronal and sagittal plane projections of the spatial vector may be similarly determined by any of the methods described.

\section{CONSTRUCTION OF THE VeCTOR LOOP}

From the Component Lead Record. "Monocardiogram" (Mann, 1920), "vector loop" (Schellong et. al., 1937) and "vectorcardiogram" (Wilson et al., 1938) are synonyms denoting a curve that connects the ends of consecutive vectors within a cardiac cycle. Visualization of a few consecutive vectors will convey the orientation of a vector loop and suggest its approximate shape (Fig. 6). If several vectors are drawn, it is preferable that they shall commence from a common point of origin. If tilted lower lead components are set off, not from the distal ends of synchronous upper lead components, but at the level of the end-points from an arbitrarily chosen common vertical line, vectors will commence from the crossing of this line with the upper lead isoelectric line.

From the Enlarged Component Lead Image. The loops in Fig. 5 and 6 were obtained with a drawing instrument (Fischmann, 1953), using photographically fourfold enlarged lead-pairs, mechanical translation of vertical shift into drawing board movement, of horizontal shift into movement of a marking point; and a scanning table larger than, but in principle similar to, that of Shillingford and Brigden (1951). The drawing instrument was used only to check the results of the visual method.

\section{FeAtures of THE LoOP ObVIOUS FROM COMPONENT LEADS, Without GeOMETRIC CONSTRUCTION OF THE LOOP}

Descriptive criteria in common use are orientation of the QRS loop; its width; orientation of " initial" and "terminal" vectors; direction of loop inscription; speed of inscription; minor irregularities of contour; failure of QRS loop to close; T loop orientation. Inspection of a component lead pair often reveals these eight criteria, without construction of single vectors, directly as does the electronic vectorcardiogram. This was suggested by a threefold approach: (1) geometric construction of lead-pair patterns from fictitious loops (summed up in Fig. 3); (2) plotting vector loops from the magnified lead-pair image with a drawing instrument (Fig. 5 and 6); (3) mathematical demonstration (Appendix).

Orientation of QRS Loop. Component lead QRS is called monophasic if the amplitude of smaller waves does not exceed one-half that of the main deflection, and diphasic if one other wave exceeds one-half that of the main deflection.

Records were made of 741 lead-pairs. In 347 lead-pairs one lead showed a mono-, the other a di-phasic QRS complex. Loops corresponding to such lead-pairs are " axial," pointed at one of the four poles of the biaxial reference system (Fig. 3). The instantaneous vector corresponding to the peak of the mono- 


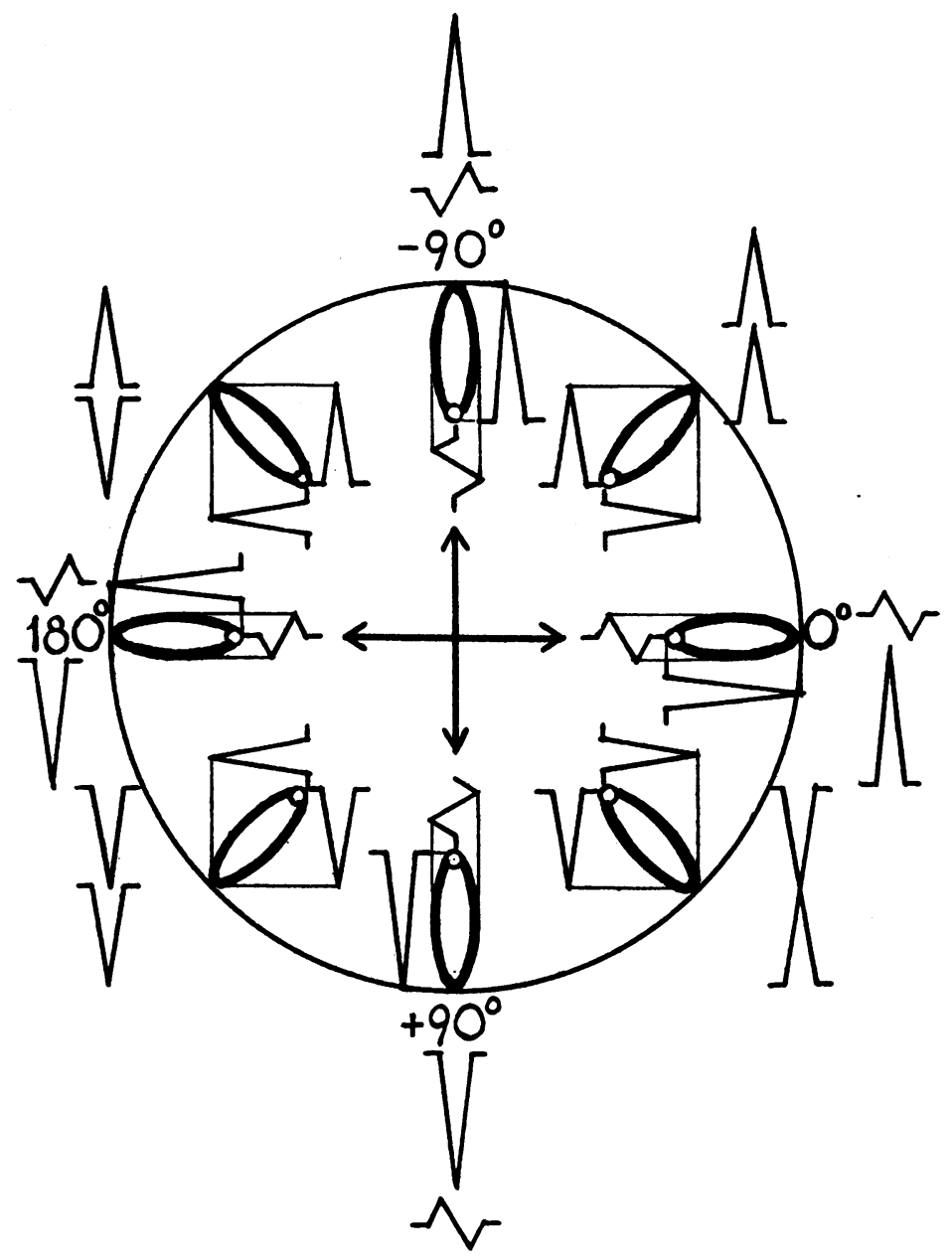

Fig. 3.-Lead-pair pattern and loop orientation. Lead-pairs as recorded by two-channel ECG, drawn outside circle; after $90^{\circ}$ clockwise tilt of lower lead drawn inside circle. Loops parallel to one of the two reference axes correspond to one monophasic and one diphasic, loops diverging from both axes to two monophasic, deflections. In axial loops, width across axis coincides with amplitude of diphasic, length along axis with that of monophasic deflection.

phasic deflection indicates loop orientation (Fig. 4A, C). Where the monophasic peak is synchronous with the isoelectric point between the two deflections of the other lead, orientation is closely along a reference axis. The loop tends to deviate from the reference axis with increasing deviation between monophasic peak and isoelectric point.

There were 316 lead-pairs showing a monophasic QRS complex in each of the two component leads. Loops corresponding to such lead-pairs are "non-axial," lying in one of the four quadrants of the biaxial reference system (Fig. 3). If the two monophasic peaks are synchronous or near-synchronous (Fig. 4B), the two peak vectors coincide, and correspond closely to the longest vector of the QRS loop. The direction of the peak vectors indicates orientation of the loop.

As discussed hereafter, lead-pairs with two monophasic deflections showing asynchronous peaks, correspond to wide loops. Most of the instantaneous vectors of a wide loop deviate from its longest vector. In such loops, especially if they are asymmetrical, the long axis is not a satisfactory indicator of orientation. Visualization of the vectors corresponding to both peaks conveys a clearer impression of loop orientation (Fig. 6). 
In the two preceding groups 78 lead-pairs could not be included, as they showed diphasic QRS in both leads, or triphasic QRS in at least one lead, or more complicated patterns. Construction or visualization of several vectors allows reconstruction of such loops. Direct prediction without vector construction is possible from some of these rarer lead-pair patterns, and will be discussed elsewhere.

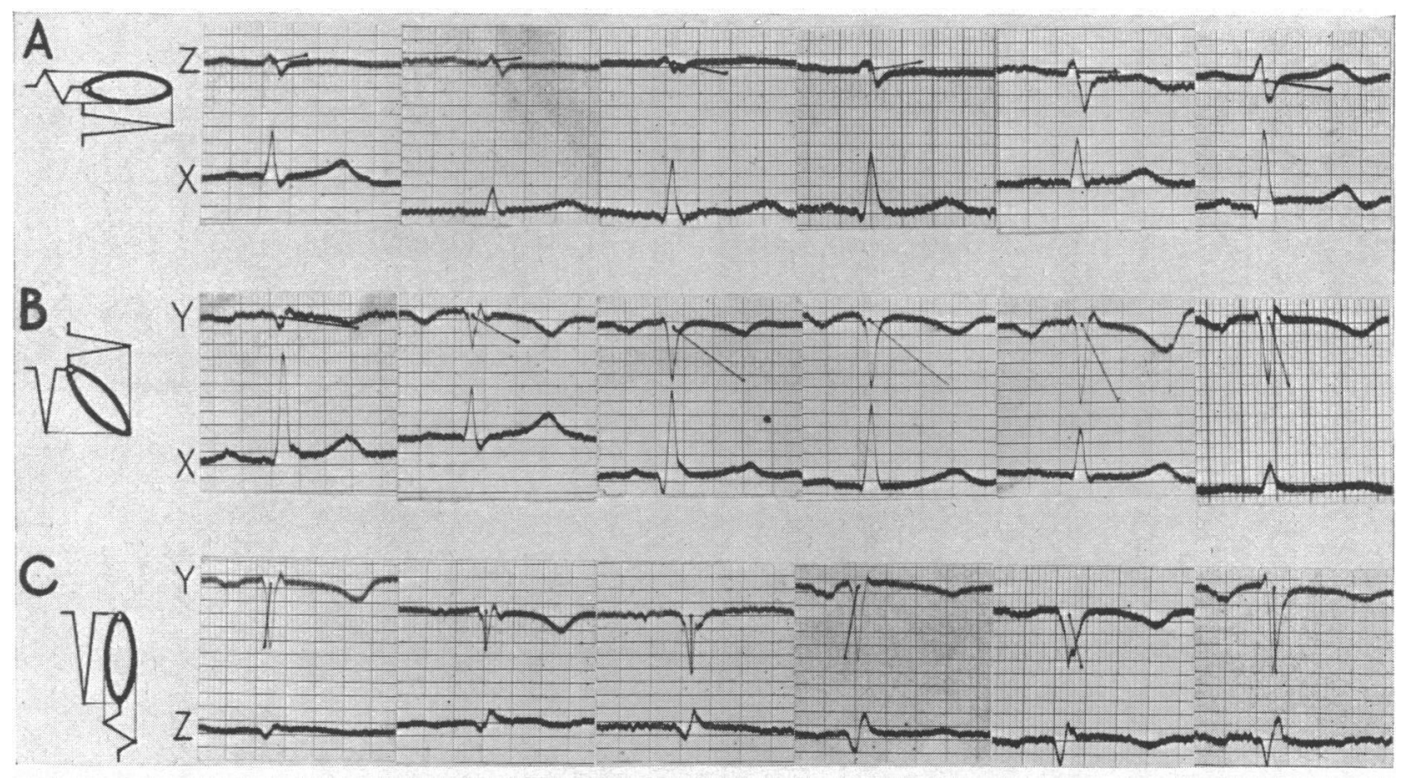

FIG. 4.-Time marks $1 / 10$ and $1 / 50 \mathrm{sec}$. (A) Coronal plane lead-pairs of 6 normal subjects. One monoand one diphasic QRS complex: " axial " QRS loops. Instantaneous vector corresponding to peak of monophasic deflection, plotted in records, indicates loop orientation. Wave succession $\mathrm{q}_{x}, 180^{\circ} ; \mathbf{R}_{z}$, $-90^{\circ} ; R_{x}, 0^{\circ} ; S_{z}, 90^{\circ}$ : clockwise inscription. Initial vectors, anteriorly (early part of $R_{z}$ ), and in varying degree to right $\left(q_{x}\right)$. (B) Frontal plane lead-pairs of 6 normal subjects. Monophasic QRS complexes in both leads: " non-axial " QRS loop. Deflection peaks synchronous or near-synchronous: narrow loops. Loop orientation indicated by instantaneous vector corresponding to tallest peak (plotted in record). Initial vectors right $\left(\mathrm{q}_{x}\right)$ and, in all but first and fourth records, also upward ( $\left.\mathrm{r}_{y}\right)$. (C) Sagittal plane lead-pairs of 6 normal subjects. One mono- and one diphasic QRS complex: " axial" QRS loops. Instantaneous vector corresponding to monophasic peak, plotted in each record, indicates orientation. Wave succession $\mathrm{Q}_{z}, 180^{\circ} ; \mathrm{S}_{y}, 90^{\circ} ; \mathrm{R}_{z}, 0^{\circ}$ : counterclockwise inscribed loops. Width of loops in (A) and $(\mathrm{C})$ increases with relative amplitude of diphasic deflections.

Width of QRS Loop. In clinical vectorcardiography it is customary to describe loops as wide or narrow without precise quantitative meaning. Width and also peak synchronism or asynchronism will be treated in this general, non-quantitative manner in the present study.

An approximate estimate of the width of " axial " loops (one mono- and diphasic lead) is obtained from recorded leads by comparing the monophasic lead amplitude with the amplitude of the diphasic lead. The loops in Fig. 5, corresponding to Fig. 4A and C, increase in width with increasing relative amplitude of the diphasic lead. Fig. 3 shows schematically that the length of a fictitious, strictly axial loop is equal to the amplitude of the monophasic component lead deflection; its width to the total amplitude of the diphasic deflection.

"Non-axial" loops (two monophasic leads), if narrow, correspond to lead-pairs where the peaks of the two leads are synchronous, or nearly synchronous (Fig. 4B); if wider, to lead-pairs with asynchronous monophasic peaks (Fig. 6). It can be shown mathematically (see Appendix) that the width of a vector loop increases with increasing asynchronism of peaks.

Initial and Terminal Vectors of QRS Loop. Small initial q, $\mathrm{r}$, and terminal $\mathrm{r}$, or s component lead waves have so far not been considered. Inspection of these waves, keeping in mind the direction they represent, will reveal the direction of the initial and terminal QRS vectors. The third lead-pair of Fig. 4B shows a small $\mathrm{r}$ in lead $\mathrm{Y}$, and a larger $\mathrm{q}$ in lead $\mathrm{X}$ : the initial vectors point to the right and slightly headward (Fig. 2, insets). 


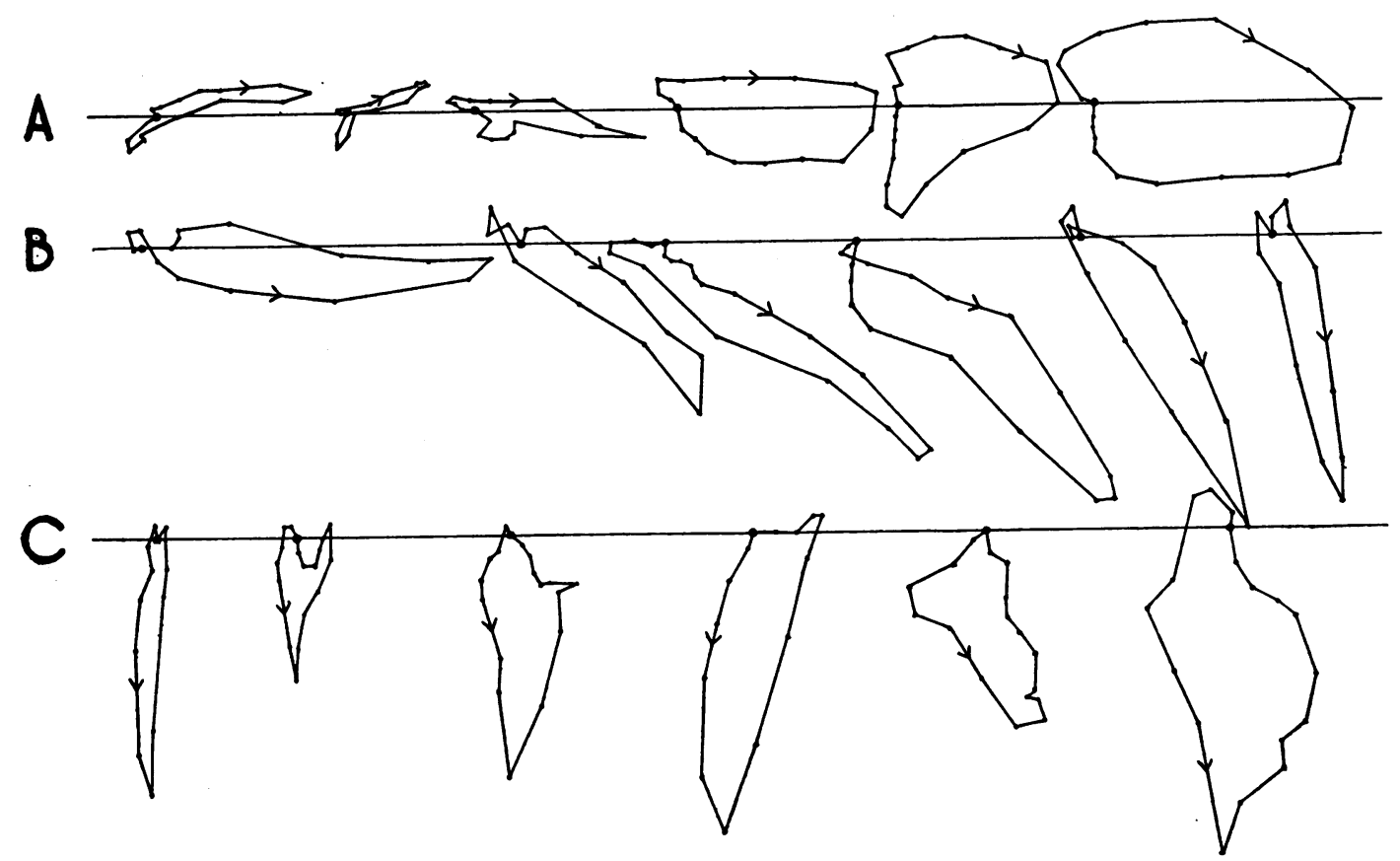

FIG. 5.-QRS loops corresponding to lead-pairs in Fig. 4; plotted with drawing instrument.

Speed of Loop Inscription. The instantaneous amplitude of a scalar cardiographic deflection depends on the magnitude of the instantaneous vector, and on the angle enclosed by the vector and the axis of the lead. In the course of vector rotation cardiographic amplitude increases to a point where axis and vector are parallel, indicated by the peak of the cardiograph deflection. Thereafter, with increasing divergence between axis and vector, amplitude decreases again. If the speed of vector rotation is constant, the scalar deflection will show gradual increase and decrease of amplitude. Transient slowing of vector rotation reduces the rate at which the amplitude of cardiographic curves changes from instant to instant. Thus in the first record of Fig. 6, synchronous rounding of $S_{z}$ and $R_{x}$ indicates slowing of loop inscription.

Direction of QRS Loop Inscription. Vectors at several subsequent instants of the cardiac cycle indicate whether the loop was inscribed clockwise or counterclockwise. This information can be obtained by noting the succession in time of component lead peaks, keeping in mind the direction represented by each peak. In all records of Fig. 4A, the peak of $Q_{x}$ is the first to appear. $\mathbf{R}_{z}$ is next, followed by $\mathbf{R}_{x}$, then $\mathbf{S}_{z}$ and in some records $S_{x}$ in that order. After rotating lead $X$ clockwise by $90^{\circ}, Q_{x}$ points to $180^{\circ}, R_{z}$ to $-90^{\circ}$, $R_{x}$ to $0^{\circ}$ and $S_{x}$ to $180^{\circ}$. A succession of $180,-90,0,90$ and 180 degrees indicates clockwise rotation. In the third record of Fig. 6 wave succession is $Q_{z} 180^{\circ}, Q_{y} 90^{\circ}, R_{z} 0^{\circ}, R_{y}-90^{\circ}$, indicating counterclockwise rotation. This approach is applicable in the majority of lead-pair records. Where QRS is a single wave without initial and terminal (q or s) deflection, the $0.02,0.03,0.04$, sec. or other consecutive time vectors, will give the direction of loop inscription (Fig. 6, first record).

Minor Irregularities of QRS Loop Contour. These are indicated by notching or splintering of the component lead curve. By visualizing an instantaneous vector which is synchronous with the notched part of the component lead curve, the irregular section of the loop can be identified.

Open QRS Loop. Instantaneous vectors of a cardiac cycle have a common point of origin. Inscription of the QRS loop commences at that point, and usually ends at it (Fig. 5). In some cases the incoming limb fails to return to the point of origin (Fig. 6). This is apparent from the component lead tracing: between $Q R S$ and $T$ there will be no instant at which the two curves are synchronously isoelectric. In the third record of Fig. 6, the descending limb of $R_{z}$ crosses the isoelectric line first, whilst lead $Y$ is still positive. By the time $Y$ reaches the isoelectric line, $Z$ has dropped below it.

Orientation of T Loop. Applying the contents of the paragraph on QRS loop orientation to the $T$ vector loop, the orientation of the latter is indicated by the vector which corresponds to the peak of the taller component lead $\mathrm{T}$ wave (Fig. 6). 

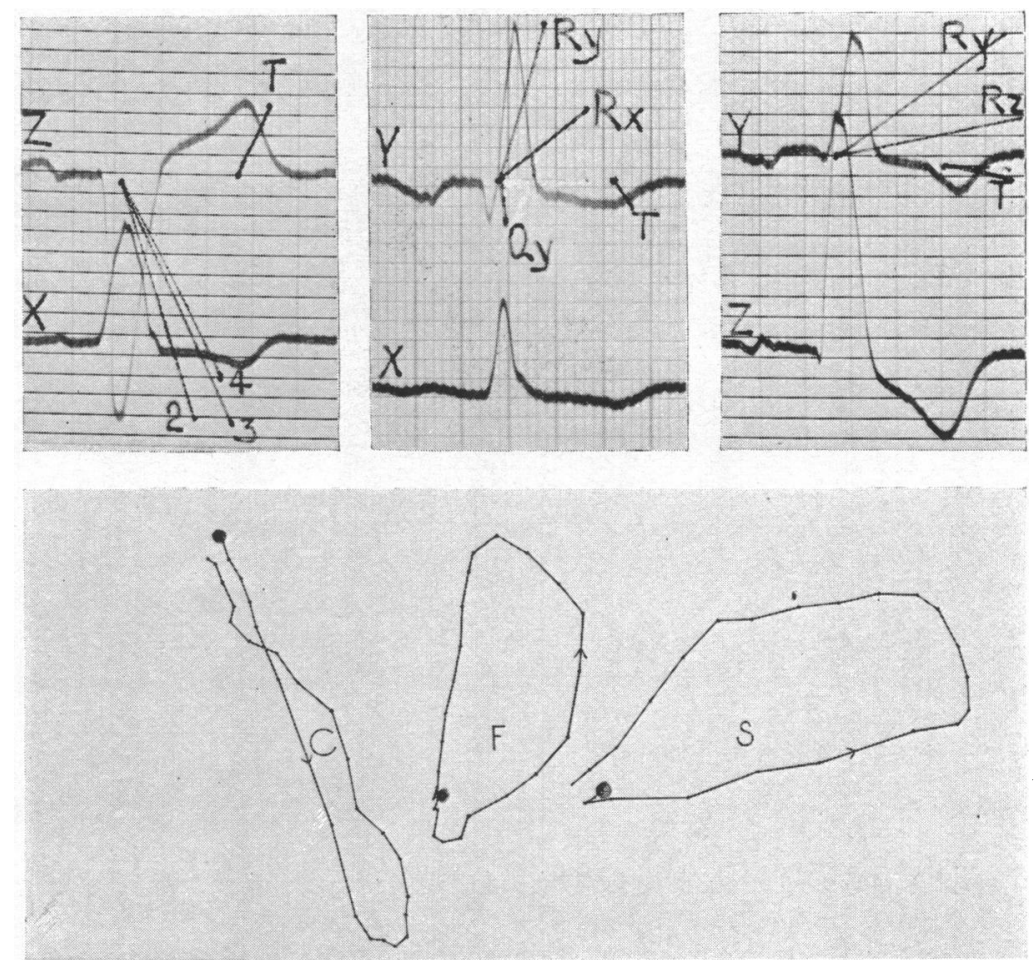

Fig. 6.-Lead-pairs with monophasic QRS complexes: "non-axial" loops. Peaks of second and third record asynchronous: wide loops. Instantaneous vectors corresponding to peaks, convey loop orientation. Synchronous rounding of $S_{z}$ and $R_{x}$ peaks in first record: slowing of loop inscription (crowded time marks, apex of plotted loop). In first and third records no instant at which both $S-T$ segments simultaneously isoelectric: "open" loops. F frontal, C coronal, S sagittal. Time marks $1 / 10$ and $1 / 50$ sec.

\section{SUMMARY}

In each of three planes a "vertical" and "horizontal" component lead was recorded by a two-channel electrocardiograph. Synchronous instantaneous amplitudes of the two curves supplied vertical and horizontal components of the instantaneous vector. The latter was visualized on the coordinate system of the cardiograph paper, using an established method of vector geometry, by setting the $90^{\circ}$ clockwise tilted lower component off from the end of the upper lead component and by joining the onset of the stable to the end of the displaced component. A biaxial reference diagram is also described, giving an alternative method of vector visualization from component leadpairs.

In the majority of cases the characteristics of the vector loop were predictable without construction of vectors, by inspection of the lead-pair record. Of the 741 lead-pairs recorded in 247 subjects 89.5 per cent showed one of two component lead-patterns: monophasic QRS in both leads, or one mono- and one diphasic QRS. The predictable loop types, corresponding to these two patterns are described.

In routine analysis prediction of the loop from the lead pair record, without geometric construction, was used first because of its directness and speed. If this did not yield all the information needed, or if the lead pair did not show one of the two common patterns, single instantaneous vectors were then determined. Photographically enlarged lead-pairs, and loops plotted therefrom 
with a drawing instrument, were finally used for purposes of the present inquiry, not as a clinical method. These techniques in their totality supplied a method, the accuracy of which was adaptable to the purpose of study.

\section{APPENDIX}

Vectorcardiographic loops are related to the well known "Lissajous" figures, used in physics and engineering for comparing the frequencies and phases of vibrations. The relationship of these figures, to the input waves from which they are integrated, is governed by strict mathematical laws. In the section on "Features of the vector loop, available without vector construction" of the preceding paper, electrocardiographic wave form was correlated with the corresponding vector loop. This was based on geometric construction of lead-pair patterns from fictitious loops and on construction of loops from enlarged cardiographic leads. The resemblance between vectorcardiograph and Lissajous loops is sufficiently close to make the essential truth of such correlation clear to the physicist. In view of the great variation and complexity of electrocardiographic wave forms, mathematical proof of general validity is impracticable here. It is fair to say, however, that mathematically demonstrating in a number of fictitious loops the correlations claimed in the quoted chapter, would tend to stress their validity. A number of loops based upon sinusoidal waves, which are easily treated mathematically, were considered from a theoretical point of view in the following. The results were in keeping with those of the geometric and empirical methods used in the preceding paper.

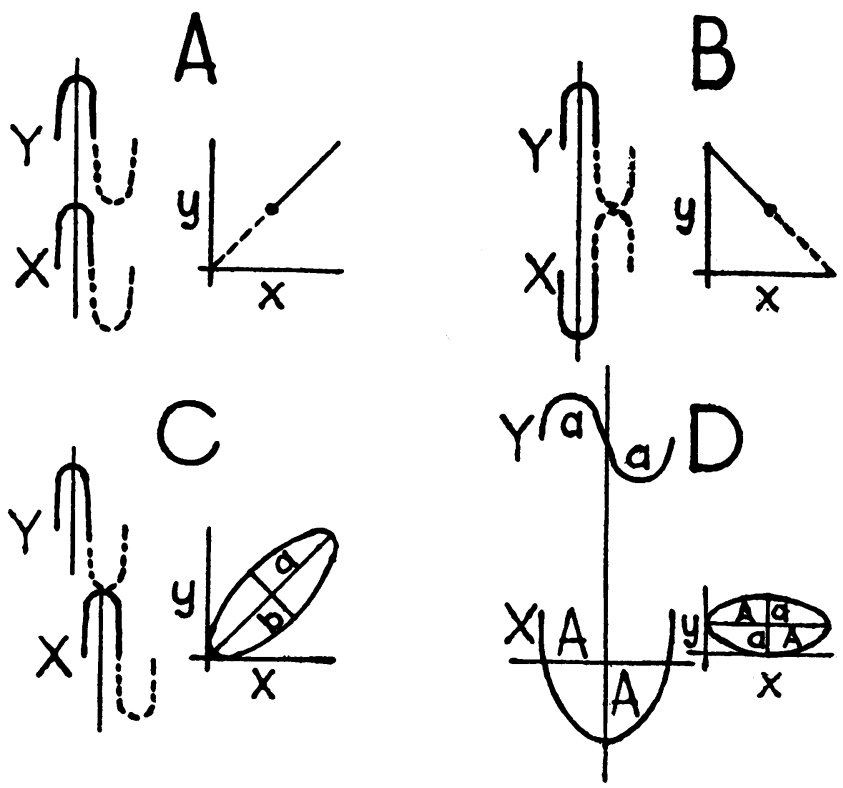

Fig. 7.-See text of Appendix.

Example $A$ (Fig. 7A). $\mathrm{X}$ and $\mathrm{Y}$ synchronous, both monophasic and upward; i.e. $\mathrm{X}$ and $\mathrm{Y}$ correspond to the first halves of two sine waves, which are in phase. The trigonometrical representations of two complete sine waves are $x=\sin t ; y=\sin t$; therefore $x=y$, which is the equation representing a straight line at $45^{\circ}$, as shown. In the present case only the first half of this line will be traced. It will point toward $-45^{\circ}$ of the reference system shown in Fig. 2. (This is to be regarded as the limiting case of a nonaxial loop, in which the width is zero.)

Example B (Fig. 7B). $\mathrm{X}$ and $\mathrm{Y}$ synchronous, both monophasic; $\mathrm{Y}$ upward, $\mathrm{X}$ downward, corresponding to one-half each of a sine wave, but $180^{\circ}$ out of phase. 
$y=\sin t ; x=-\sin t ;$ therefore $x=-y$. This is the equation of a straight line at $-45^{\circ}$, as shown. Only the first half of this line will be traced. It will point toward $-135^{\circ}$ of the reference system in Fig. 2 (Another infinitely narrow nonaxial loop).

Example $C$ (Fig. 7C). $\mathrm{X}$ and $\mathrm{Y}$ both monophasic and upward; asynchronous, e.g. suppose $\mathrm{Y}$ to be ahead of $\mathrm{X}$ by an interval of time $\epsilon$.

$$
y=\sin (t+\epsilon) ; x=\sin t
$$

expanding the equation for $y$,

$$
y=\sin t \cos \epsilon+\cos t \sin \epsilon
$$

And re-writing the expression for $x$ as $\sqrt{1-x^{2}}=\cos t$,

then

$$
\begin{gathered}
y=x \cos \epsilon+\sqrt{1-x^{2}} \sin \epsilon \\
\left(1-x^{2}\right) \sin ^{2} \epsilon=(y-x \cos \epsilon)^{2}, \\
y^{2}+x^{2}-2 y x \cos \epsilon=\sin ^{2} \epsilon .
\end{gathered}
$$

or

This is an equation representing an ellipse as shown, the principal axes $a$ and $b$ being at $45^{\circ}$ and $-45^{\circ}$ to the $X$ axis respectively. The lengths of the axes are in a ratio given by: $\frac{b^{2}}{a^{2}}=\frac{1-\cos \epsilon}{1+\cos \epsilon}$; whence $\frac{b}{a}=\tan \frac{\epsilon}{2}$ numerically. The eccentricity therefore depends on the time interval $\epsilon$. Nearly synchronous pulses have small values of $\epsilon$ and hence narrow ellipses. The ellipse here is traced in a clockwise direction and in the case chosen only its first half will be inscribed. The waves are out of phase at zero level: the loop will remain " open." In a strictly similar way, we can show that if $Y$ is lagging behind $X$ by an interval of time, the ellipse is similar in appearance but is described in a counterclockwise direction.

Example D (Fig. 7D). X monophasic downward, Y diphasic. The peak of the monophasic lead is taken as synchronous with the isoelectric centre between the two peaks of the diphasic curve. In the previous examples it was assumed for simplicity that the amplitudes of the $\mathrm{X}$ and $\mathrm{Y}$ curves were equal, but now we will make a more general assumption, viz., let $A=$ amplitude of monophasic wave; $a=$ amplitude of diphasic wave, where $a$ is less than $A$. (With the sinusoidal form which we are assuming for the waves, an amplitude $A$ will imply a total pulse-height $2 A$ for the monophasic wave.) $y=a \sin t ; x=A \cos t$; $\operatorname{since} \sin ^{2} t+\cos ^{2} t=1$; $\frac{y^{2}}{a^{2}}+\frac{x^{2}}{A^{2}}=1$, which is the equation describing an axial ellipse or loop as shown, with the longer axis of the loop horizontal. In an exactly similar way we can see that if the monophasic and diphasic waves are interchanged, the resulting loop will have its longer axis vertical instead of horizontal.

Expressed in the terms used in the preceding paper: A lead-pair with two synchronous monophasic upward deflections corresponds to a narrow nonaxial loop in the fourth quadrant (A). A lead-pair with synchronous upward monophasic upper lead, and downward monophasic lower lead deflections, corresponds to a narrow nonaxial loop in the third quadrant (B). A lead-pair with two asynchronous monophasic upward deflections corresponds to a wide non-axial loop in the fourth quadrant. Width increases with asynchronism. Direction of inscription depends on peak succession (C). A lead-pair with upper lead diphasic, and lower lead downward monophasic deflection, corresponds to an axial loop, orientated toward $180^{\circ}(\mathrm{D})$.

\section{REFERENCES}

Duchosal, P. W., and Sulzer, R. (1949) La Vectocadiographie. Basle.

Fischmann, E. J. To be published.

Graettinger, J. S., Packard, J. M., and Graybiel, A. (1951). Amer. J. Med., 11, 3.

Grant, R. P., and Estes, E. H., Jr. (1951). Spatial Vector Electrocardiography. Philadelphia.

Graybiel, A., and White, P. D. (1952). Electrocardiography in Practice. Philadelphia.

Grishman, A., Borun, E. E., and Jaffe, H. L. (1951). Amer. Heart J., 41, 483.

Henrici, O., and Turner, G. C. (1903). Vectors and Rotors. London.

Holzmann, M. (1945). Klinische Electrokardiographie. Zurich.

Lepeschkin, E. (1951). Modern Electrocardiography, Vol. 1. Baltimore.

Mann, H. (1920). Arch. intern. Med., 25, 283.

Rutherford, D. E. (1951). Vector Methods. Edinburgh.

Shillingford, J., and Brigden, W. (1951). Brit. Heart J., 13, 233.

Wenkebach and Winterberg (1927). Quoted from Holzmann (1945), p. 60.

Wilson, F. N., and Johnston, F. D. (1938). Amer. Heart .J., 16, 14. 